\title{
XÁC ĐỊNH ĐẠI LƯợNG ĐặC TRƯNG ĐỘ TIN CẬY CỦA ĐƯỜNG CHUYỀN
}

\author{
PGS. TS. TRƯO'NG QUANG HIÉU \\ ThS. LÊ NGOQC GIANG \\ Trường Đại học Mỏ - Địa chất
}

\section{Tóm tắt:}

Bài báo đã đề xuất sử dụng tỷ số $G(p)$ để giảm khối lượng tính đại lượng đặc trưng độ tin cậy của đường chuyền.

\section{1. Đặt vấn đề}

Trong các tài liệu [1], [2] các tác giả đã đề xuất sử dụng đại lượng $\sqrt[p]{\operatorname{det}_{X}}$ của ma trận hiệp phương sai tọa độ $\mathrm{P}$ điểm cần tìm trong đường chuyền làm đại lượng đặc trưng cho độ tin cậy của đường chuyền đó.

Trong thực tế đối với lưới khống chế trắc địa mặt bằng sai số vị trí điểm hay diện tích các đường cong đặc trưng cho sai số vị trí điểm cần tìm lại có ý nghĩa rất quan trọng. Trong trường hợp này sử dụng dạng gần đúng ma trận $M_{X}^{0}$ - gồm các phần tử chứa trong các ma trận cấp $(2 \times 2)$ là $M_{X(2 \times 2)}^{(u)}$ (tương ứng của các tọa độ $\mathrm{X}$ và $\mathrm{Y}$ của điểm thứ $(\mathrm{u})$ ) kế tiếp trên đường chéo chinh của ma trận $M_{X}$ thay thế cho ma trận $M_{X}$ và dùng đại lượng $\sqrt[p]{\operatorname{det} M_{X}^{0}}$ thay thế đại lượng $\sqrt[p]{\operatorname{det} M_{X}}$ để đánh giá độ tin cậy của lưới. Từ đó ta có quan hệ

$$
\operatorname{det}_{X}^{0}=\prod_{u=1}^{p} \operatorname{det} M_{X(2 \times 2)}^{u}
$$

Từ quan hệ giữa các giá trị định thức với diện tích của elip sai số vị trí điểm thứ (u) có các bán trục $A, B$ (tài liệu [2])

$$
S_{E}^{u}=\pi A B=\pi \sqrt{\operatorname{det} M_{X(2 \times 2)}^{u}}
$$

Ta viết (1) dưới dạng:

$$
\operatorname{det}_{X}^{0}=\frac{\prod_{n=1}^{p} S_{E}^{u}}{\pi^{p}}
$$

Hay

$$
\sqrt[p]{\operatorname{det}_{X}}=\sqrt[p]{\operatorname{det} M_{X}^{0}}=\frac{\sqrt[p]{\prod_{u=1}^{p} S_{E}^{(u)}}}{\pi}=\frac{S_{E}}{\pi}
$$

Trong (3) đại lượng $\overrightarrow{\mathbf{s}_{\mathbb{F}}}$ được ký hiệu là trị trung bình nhân diện tích elip sai số vị trí $p$ điểm cần tìm trong đường chuyền.

Từ (3) ta thấy có thể dùng đại lượng $\sqrt[p]{\operatorname{det} M_{X}}$ hay đại lượng $\bar{S}_{\mathbb{F}_{2}}$ làm hàm mục tiêu để đánh giá độ tin cậy của lưới khống chế trắc địa mặt bằng nói chung và lưới đường chuyền nói riêng.

Người phản biện: TS. Dương Chí Công 
Do đặc điểm của đường chuyền, nên chúng ta có thể xác định các đại lượng $\overrightarrow{s_{\mathbb{R}}}$ hay $\sqrt[p]{\operatorname{det} M_{X}}$ thông qua diện tích của đường cong sai số vị trí điểm giữa và hệ số tỷ lệ:

$$
G_{(p)}=\frac{S_{E}^{\text {gita }}}{\overline{S_{E}}}
$$

Tham số $p$ trong $G_{(p)}$ chỉ số lượng điểm cần tìm của đường chuyền.

Khi sử dụng công thức này sẽ giảm khối lượng tính đại lượng đặc trưng độ tin cậy của đường chuyền đi rất nhiều. Chúng ta không cần phải tính trị trung bình nhân của diện tích các đường tròn (hay elip) sai số vị trí của $p$ điểm cần tìm trong đường chuyền, mà chỉ cần tìm diện tích của đường tròn (hay elip) sai số vị trí điểm giữa tuyến.

\section{Giải quyết vấn đề}

Đường chuyền lý tưởng (duỗi thẳng, cạnh đều) đo 2 góc nối là đường chuyền có độ tin cậy cao nhất trong các đường chuyền có cùng số lượng điểm cần tìm $(p)$ và chiều dài cạnh trung bình tương đương. Dựa vào tính chất của sai số vị trí điểm của đường chuyền đo 2 góc nối chúng ta có thể xác định trị lý thuyết của tỷ số $\mathrm{G}_{(\mathrm{p})}$ giữa diện tích đường tròn sai số vị trí điểm giữa với trị trung bình nhân diện tích đường tròn sai số của $p$ điểm trong đường chuyền. Để đạt được mục tiêu trên ta sử dụng công thức của $\mathrm{G}$. $\mathrm{S}$. Hausbrandt chứng minh trong [4] cho trường hợp đường chuyền lý tưởng đo 2 góc nối có $(p)$ điểm cần tìm và có hướng trùng với trục $O X$ trong hệ tọa độ (XOY). Với các giả thiết các góc đo cùng độ chính xác là $\frac{m_{\beta}}{\rho^{*}}$; các cạnh đo cùng độ chính xác $\frac{m_{s}}{S}$ và chiều dài cạnh bằng $\mathrm{S}$, sau khi bình sai sẽ thu được trị bình sai của sai số dịch vị ngang $M_{n g}^{*}$ và dịch vị dọc $M_{d}^{*}$ dạng:

$$
M_{n g}^{*}=\frac{m_{\beta}}{\rho^{\prime \prime}} \cdot S \cdot \sqrt{\frac{i(p-i+1)(p-i+2)(i+1)}{6(p+1)(p+2)(p+3)} \cdot\left[(2 \mathrm{i}+1)-\left(2 \mathrm{i}^{2}-2 \mathrm{i}-3\right)\right]}
$$

Và

$$
M_{d}^{*}=\frac{m_{s}}{S} \cdot S \cdot \sqrt{\frac{i(p-i+1)}{(p+1)}}
$$

Trong đó $i=1 \div p$

Đặt

$$
\begin{aligned}
M_{n g}^{*(i)} & =\frac{m_{\beta}}{\rho^{\prime \prime}} \cdot S \cdot \bar{M}_{n g}^{(i)} \\
M_{d}^{*(i)} & =\frac{m_{s}}{s} \cdot S \cdot \bar{M}_{d}^{(i)}
\end{aligned}
$$

Trong các công thức (5), (6) i là chỉ số của vị trí điểm cần tìm trong đường chuyền. Sử dụng công thức (5), (6) ta tìm được sai số dịch vị ngang, dịch vị dọc của các điểm cần tìm trong các đường chuyền có $p=5$ điểm, $p=7$ điểm, $p=9$ điểm cần tìm. Kết quả ghi ở bảng 1. 
Với

$$
\begin{gathered}
\bar{M}_{n g}^{(i)}=\sqrt{\frac{i(p-i+1)(p-i+2)(i+1)}{6(p+1)(p+2)(p+3)} \cdot\left[(2 \mathrm{i}+1)-\left(2 \mathrm{i}^{2}-2 \mathrm{i}-3\right)\right]} \\
\bar{M}_{d}^{(i)}=\sqrt{\frac{i(p-i+1)}{(p+1)}}
\end{gathered}
$$

\begin{tabular}{|c|c|c|c|c|c|c|c|c|c|c|}
\hline$P$ & $\begin{array}{l}\bar{M}_{n g}^{(i)} \\
\bar{M}_{d}^{(i)}\end{array}$ & 1 & 2 & 3 & 4 & 5 & 6 & 7 & 8 & 9 \\
\hline 5 & $\begin{array}{l}0.732 \\
0.913\end{array}$ & $\begin{array}{l}1,195 \\
1,155\end{array}$ & $\begin{array}{l}1,195 \\
1,155\end{array}$ & $\begin{array}{l}1,363 \\
1,225\end{array}$ & $\begin{array}{l}1,195 \\
1,155\end{array}$ & $\begin{array}{l}0,732 \\
0,913\end{array}$ & & & & \\
\hline 7 & $\begin{array}{l}0.789 \\
0.936\end{array}$ & $\begin{array}{l}1,409 \\
1,225\end{array}$ & $\begin{array}{l}1,409 \\
1,225\end{array}$ & $\begin{array}{l}1,827 \\
1,369\end{array}$ & $\begin{array}{l}1,973 \\
1,414\end{array}$ & $\begin{array}{l}1,827 \\
1,369\end{array}$ & $\begin{array}{l}1,409 \\
1,225\end{array}$ & $\begin{array}{l}0,789 \\
0,936\end{array}$ & & \\
\hline 9 & $\begin{array}{l}0.826 \\
0.949\end{array}$ & $\begin{array}{l}1,550 \\
1,265\end{array}$ & $\begin{array}{l}1,550 \\
1,265\end{array}$ & $\begin{array}{l}2,143 \\
1,449\end{array}$ & $\begin{array}{l}2,526 \\
1,549\end{array}$ & $\begin{array}{l}2,659 \\
1,581\end{array}$ & $\begin{array}{l}2,526 \\
1,549\end{array}$ & $\begin{array}{l}2.143 \\
1.449\end{array}$ & $\begin{array}{l}1.550 \\
1.265\end{array}$ & $\begin{array}{l}0.826 \\
0.949\end{array}$ \\
\hline
\end{tabular}

Bảng 1: Kết quả tính các đại lượng $\bar{M}_{n g}^{(i)} ; \bar{M}_{d}^{(i)}$ theo công thức Hausbrandt

Trong khuôn khổ bài báo này chúng tôi chỉ xét trường hợp có $\frac{m_{\beta}}{\rho^{\prime \prime}}=\frac{m_{S}}{S}=\frac{1}{T}$

Dựa vào các sai số dịch vị ngang và dịch vị dọc của GS.Hausbrandt chúng ta chỉ tính được diện tích đường tròn sai số vị trí các điểm cần tìm. Ta có với điểm thứ (i) diện tích đường tròn sai số có dạng:

$$
S_{D T}^{(i)}=\pi \cdot R^{2^{*}(i)}=\pi \cdot\left\{\left(M_{n g}^{*(i)}\right)^{2}+\left(M_{d}^{*(i)}\right)^{2}\right\}=\pi \cdot\left(\frac{1}{T} \cdot S\right)^{2}\left\{\left(\bar{M}_{n g}^{(i)}\right)^{2}+\left(\bar{M}_{d}^{(i)}\right)^{2}\right\}
$$

Nếu nhận $M_{n g}^{*(1)}$ hoặc $\bar{M}_{n g}^{(1)}$ làm tham số thì để sử dụng công thức (9) ta tạo các tỷ số:

$$
\begin{array}{ll} 
& \frac{M_{n g}^{*(i)}}{M_{n g}^{*(1)}}=\frac{\bar{M}_{n g}^{(i)}}{\bar{M}_{n g}^{(1)}}=a_{i} \\
\text { Hay } & \bar{M}_{n g}^{(i)}=a_{i} \cdot \bar{M}_{n g}^{(1)} \\
\text { Và } & \frac{M_{d}^{*(i)}}{M_{n g}^{*(1)}}=\frac{\bar{M}_{d}^{(i)}}{\bar{M}_{n g}^{(1)}}=g_{i} \\
\text { Hay } & \bar{M}_{d}^{(i)}=g_{i} \cdot \bar{M}_{n g}^{(1)}
\end{array}
$$


Lúc này diện tích (9) sẽ có dạng

$$
\begin{aligned}
S_{D T}^{(i)}= & \pi \cdot\left(\frac{1}{T} \cdot S\right)^{2}\left[a_{i}^{2} \bar{M}_{n g}^{(1)^{2}}+g_{i}^{2} \bar{M}_{n g}^{(1)^{2}}\right]=\pi \cdot\left(\frac{1}{T} \cdot S\right)^{2} \cdot \bar{M}_{n g}^{(1)^{2}}\left[a_{i}^{2}+g_{i}^{2}\right] \\
& \pi \cdot\left(\frac{1}{T} \cdot S\right)^{2} \cdot \bar{M}_{n g}^{(1)^{2}}=\pi \cdot\left(\frac{1}{T} \cdot S\right)^{2} \cdot \bar{M}_{n g}^{(1)^{2}} \cdot U_{i}^{2}
\end{aligned}
$$

Trong (10) đại lượng $U_{i}^{2}$ được ký hiệu là:

$$
U_{i}^{2}=a_{i}^{2}+g_{i}^{2}
$$

Trong bảng 2 là kết quả tính các đại lượng $\mathrm{a}_{\mathrm{i}}, \mathrm{g}_{\mathrm{i}}$ và $U_{i}^{2}$ với đường chuyền có 9 điểm cần tìm có $\bar{M}_{n g}^{(1)}$ là tham số.

Bảng 2: Tính các giá trị $a_{i}, g_{i}$ và $U_{i}^{2}$ với $p=9$ điểm

\begin{tabular}{|c|c|c|c|c|c|c|c|c|c|}
\hline Giềm & 1 & 2 & 3 & 4 & 5 & 6 & 7 & 8 & 9 \\
\hline $\mathrm{a}_{\mathrm{i}}$ & 1,0 & 1,87 & 2,58 & 3,04 & 3,20 & 3,04 & 2,58 & 1,87 & 1,0 \\
\hline $\mathrm{g}_{\mathrm{i}}$ & 1,14 & 1,52 & 1,74 & 1,87 & 1,90 & 1,87 & 1,74 & 1,52 & 1,14 \\
\hline$U_{i}^{2}$ & 2,30 & 5,81 & 9,68 & 12,74 & 13,85 & 12,74 & 9,68 & 5,81 & 2,30 \\
\hline
\end{tabular}

Từ các kết quả trên ta tính được trị trung bình nhân của diện tích đường tròn sai số của 9 điểm cần tìm.

$$
S_{D T}^{(i)}=\pi \cdot\left(\frac{1}{T} \cdot S\right)^{2} \cdot \bar{M}_{n g}^{(1)^{2}} \cdot \sqrt[9]{2,30^{2} \times 5,81^{2} \times 9,68^{2} \times 12,74^{2} \times 13,85}=\pi \cdot\left(\frac{1}{T} \cdot S\right)^{2} \cdot \bar{M}_{n g}^{(1)^{2}} \times 6,95
$$

Và diện tích đường tròn sai số vị trí điểm giữa:

$$
S_{D T}^{G}=\pi \cdot\left(\frac{1}{T} \cdot S\right)^{2} \cdot \bar{M}_{n g}^{(1)^{2}} \times 13,85
$$

Từ đó ta tính được hệ số:

$$
G_{(p)}=G_{9}=\frac{S_{D T}^{G}}{\bar{S}_{D T}}=\frac{13,85}{6,95} \approx 1,99
$$

Ta thấy khi tỷ số $\mathrm{G}_{9}=1,99$ được kiểm chứng là chính xác thì để tính đại lượng $\bar{S}_{D T}$ hay $\left(\sqrt[9]{\operatorname{det}_{X}^{0}}\right)$ chúng ta dùng công thức:

$$
\bar{S}_{D T}=\frac{S_{D T}^{G}}{1,99}
$$


Nghĩa là chỉ cần tìm diện tích đường tròn sai số vị trí điểm giữa tuyến. Dễ nhận thấy tỷ số phụ thuộc vào số điểm cần tìm trong đường chuyền. Tính các giá trị này với số lượng điểm cần tìm cụ thể tiến hành tương tự với đường chuyền có 9 điểm cần tìm.

Nếu sử dụng các dữ liệu thu được từ kết quả bình sai để tính các sai số dịch vị dọc, dịch vị ngang và các bán trục $A, B$ của elip sai số vị trí điểm $(u)$ thì:

$$
S_{D T}^{(u)}=\pi \cdot\left(\left(M_{n g}^{*(u)}\right)^{2}+\left(M_{d}^{*(u)}\right)^{2}\right\}=\pi\left[A^{2}+B^{2}\right]
$$

Nên các tỷ số $G_{(p)}$ tính theo diện tích đường tròn sai số cũng gần giống khi tính $G_{(p)}$ theo diện tích elip sai số vị trí điểm.

\section{Tính toán thực nghiệm}

Để kiểm chứng kết quả tính trị trung bình nhân diện tích e lip sai số vị trí 9 điểm cần tìm chúng tôi tiến hành thực nghiệm với đường chuyền đo 2 góc nối trên hình 1 . Số liệu đo thực tế và số liệu gốc ghi trong các bảng 3 và 4 . (Xem hình 1, bảng 3, 4).

Tiến hành bình sai lưới đường chuyền bằng phương pháp bình sai gián tiếp chặt chẽ. Kết quả tính toán từng bước cụ thể trình bày trong tài liệu [3]. Dưới đây chúng tôi trích lược những kết quả cần thiết phục vụ cho việc kiểm chứng các đại lượng $\bar{S}_{E}$ hay $\mathrm{G}_{(9)}$. (Xem bảng 5, 6).

Từ kết quả ở bảng 6 ta thu được $G_{(9)}=1,90$. So với tỷ số lý thuyết $G_{(9)}=1,99$, thì tỷ số thực tế này chênh nhau $9 \%$ tương đương với $\mathrm{c} \times 0,09=0,5$ là số chênh không đáng kể, khi sai số vị trí điểm cho phép theo quy phạm thường từ $(4 \div 5) \mathrm{cm}^{2}$.

\section{Kết luận}

Từ nội dung trình bày trong bài báo chúng tôi rút ra một số kết luận:

1. Đề xuất sử dụng trị trung bình nhân $\bar{S}_{E}$ của diện tích elip sai số vị trí các điểm cần tìm trong đường chuyền làm đại lượng đặc trưng cho độ tin cậy của đường chuyền.

2. Nên sử dụng tỷ số $\mathrm{G}_{(\mathrm{p})}$ để giảm khối lượng tính đại lượng đặc trưng $\bar{S}_{E}$. Chúng ta có thể tính sẵn tỷ số $\mathrm{G}_{(\mathfrak{p})}$ tương ứng với sự thay đổi của số điểm cần tìm trong đường chuyền thì sẽ nâng đáng kể hiệu quả sử dụng tỷ số này.

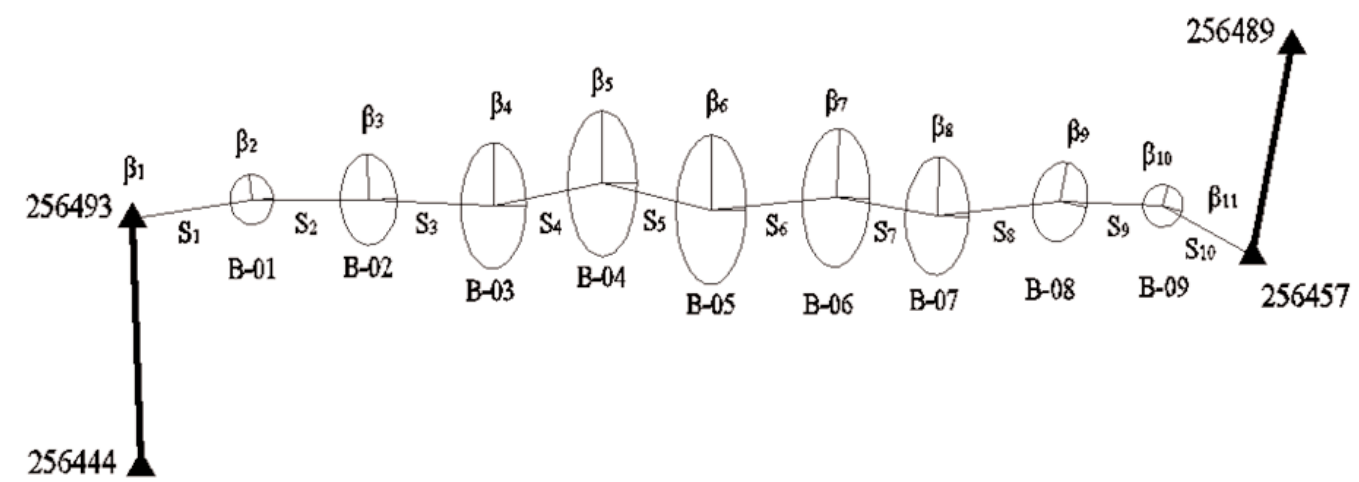

Hình 1: Sơ đồ lưới 
Bảng 3: Số liệu gốc

\begin{tabular}{|c|c|c|}
\hline Tên điểm & $\mathbf{X}(\mathbf{m})$ & $\mathbf{Y}(\mathbf{m})$ \\
\hline 256444 & 2032990,730 & 510342,291 \\
\hline 256493 & 2034747,081 & 510280,070 \\
\hline 256457 & 2034490,820 & 518207,980 \\
\hline 256489 & 2036345,450 & 518560,020 \\
\hline
\end{tabular}

Bảng 4: Số liệu đo

\begin{tabular}{|c|c|c|c|}
\hline TT góc & Giá trị góc ( $^{\circ}{ }^{\prime}$ “) & TT cạnh & Giá trị cạnh $(\mathbf{m})$ \\
\hline$\beta 1$ & 2630027 & $\mathrm{~S}_{1}$ & 855,968 \\
\hline$\beta 2$ & 1890117 & $\mathrm{~S}_{2}$ & 827,530 \\
\hline$\beta 3$ & 1824613 & $\mathrm{~S}_{3}$ & 885,632 \\
\hline$\beta 4$ & 1654345 & $\mathrm{~S}_{4}$ & 786,254 \\
\hline$\beta 5$ & 2050138 & $\mathrm{~S}_{5}$ & 792,432 \\
\hline$\beta 6$ & 1605713 & $\mathrm{~S}_{6}$ & 888,705 \\
\hline$\beta 7$ & 1954305 & $\mathrm{~S}_{7}$ & 724,835 \\
\hline$\beta 8$ & 1631550 & $\mathrm{~S}_{8}$ & 876,050 \\
\hline$\beta 9$ & 1884704 & $\mathrm{~S}_{9}$ & 728,209 \\
\hline$\beta 10$ & 2063105 & $\mathrm{~S}_{10}$ & 722,656 \\
\hline$\beta 11$ & 715910 & & \\
\hline
\end{tabular}

Bảng 5: Tính toạ độ sau bình sai

\begin{tabular}{|c|c|c|c|c|c|c|}
\hline \multirow{2}{*}{$\begin{array}{c}\text { TT } \\
\text { điểm }\end{array}$} & \multicolumn{2}{|c|}{ Toạ độ gần đúng } & \multicolumn{2}{c|}{ Số hiệu chỉnh } & \multicolumn{2}{c|}{ Toạ độ sau bình sai } \\
\cline { 2 - 7 } & $\mathbf{X}(\mathbf{m})$ & $\mathbf{Y}(\mathbf{m})$ & $\Delta \mathbf{X}(\mathbf{m m})$ & $\Delta \mathbf{Y}(\mathbf{m m})$ & $\mathbf{X}(\mathbf{m})$ & $\mathbf{Y}(\mathbf{m})$ \\
\hline $\mathbf{1}$ & 2034881,316 & 5511125,450 & -7 & -1 & 2034881,309 & 511125,449 \\
\hline $\mathbf{2}$ & 2034881,336 & 511952,982 & -9 & -1 & 2034881,327 & 511952,981 \\
\hline $\mathbf{3}$ & 2034838,558 & 512837,581 & -8 & -1 & 2034838,550 & 512837,580 \\
\hline $\mathbf{4}$ & 2034995,345 & 513608,048 & -5 & -4 & 2034995,340 & 513608,044 \\
\hline $\mathbf{5}$ & 2034810,027 & 514378,505 & -1 & 0 & 2034810,026 & 514378,504 \\
\hline $\mathbf{6}$ & 2034895,548 & 515263,088 & 4 & -2 & 2034895,552 & 515263,086 \\
\hline $\mathbf{7}$ & 2034767,246 & 515976,477 & 6 & 0 & 2034767,252 & 515976,477 \\
\hline $\mathbf{8}$ & 2034867,037 & 516846,828 & 8 & -2 & 2034867,045 & 516846,826 \\
\hline $\mathbf{9}$ & 2034838,532 & 517574,480 & 6 & -2 & 2034838,538 & 517574,478 \\
\hline
\end{tabular}


Bảng 6: Tính diện tích elip sai số và kiểm chứng kết quả tính tỷ số G(9)

\begin{tabular}{|c|c|c|c|c|c|}
\hline TT & $\varphi_{A}\left({ }^{\circ}, "\right)$ & $\mathrm{A}(\mathrm{cm})$ & $\mathrm{B}(\mathrm{cm})$ & $8_{E^{3}} p^{3 / 3}\left(\mathrm{~cm}^{2}\right)$ & $\sqrt{\operatorname{det} M_{X}}$ \\
\hline 1 & -43950 & 0,9 & 0,8 & 2,1 & 0,7 \\
\hline 2 & -14306 & 1,6 & 1,0 & 5,0 & 1,6 \\
\hline 3 & 05609 & 2,2 & 1,1 & 8,1 & 2,6 \\
\hline 4 & -02151 & 2,6 & 1,2 & 9,8 & 3,1 \\
\hline 5 & 04341 & 2,7 & 1,2 & 10,3 & 3,3 \\
\hline 6 & 22436 & 2,5 & 1,2 & 9,2 & 2,9 \\
\hline 7 & 20400 & 2,1 & 1,1 & 7,3 & 2,3 \\
\hline 8 & 100657 & 1,4 & 0,9 & 4,2 & 1,3 \\
\hline 9 & 141546 & 0,8 & 0,7 & 1,7 & 0,5 \\
\hline$\prod_{g} S_{z}^{(x)}$ & & & & 4112958,33 & 137,98 \\
\hline $\bar{S}_{E}$ & & & & 5,43 & 1,73 \\
\hline $\mathrm{G}_{(9)}$ & & & & 1,90 & \\
\hline$\sqrt{\operatorname{det} M_{X}}$ & & & & 1,73 & \\
\hline
\end{tabular}

Tài liệu tham khảo

[1]. Trương Quang Hiếu, Lưu Anh Tuấn. Cơ sở toán học của lý thuyết sai số và phương pháp bình sai trắc địa. NXB Giao thông vận tải. Hà Nội, 2014.

[2]. Nguyễn Việt Hải Linh. Đánh giá độ tin cậy đường chuyền thông qua đại lượng đặc trưng cho elip sai số vị trí điểm. Luận văn Thạc sĩ Kỹ thuật. Đại học Mỏ địa chất, 2014.

[3].GS.Hausbrandt, Rachunek Wyrównawczy Obliczenia geodezyjne. PPWK WARZAWA, 1971.0

\section{Summary}

\section{Determining the reliability ratio of traverse}

Assoc. Prof. Dr. Truong Quang Hieu, MSc. Le Ngoc Giang

University of Mining and Geology

This paper has proposed using the ratio $G_{(p)}$ to reduce the amount of needed work to calculate the reliability ratio of traverse. Thereby, the ratio $G_{(p)}$ can be calculated in advance, corresponding to the change of some points which need to be found in the traverse. This will improve efficiency of using the ratio. $\bigcirc$

Ngày nhận bài: 10/12/2014. 